\title{
Patients who leave the emergency department against medical advice
}

eISSN: 2383-4625

\section{Choung Ah Lee', Joon Pil Cho², Sang Cheon Choi ${ }^{2}$, Hyuk Hoon Kim², Ju Ok Park ${ }^{1}$}

'Department of Emergency Medicine, Hallym University Dongtan Sacred Heart Hospital, Hwaseong, Korea ${ }^{2}$ Department of Emergency Medicine, Ajou University School of Medicine, Suwon, Korea

Objective Discharge against medical advice (DAMA) from the emergency department (ED) accounts for $0.1 \%$ to $2.7 \%$ of all ED discharges. DAMA carries a risk of increased mortality and readmissions. Our aim was to investigate the general characteristics of DAMA patients and the differences between them and non-DAMA patients.

Methods We reviewed data collected by the National Emergency Medical Center between 2010 and 2011. Subjects were categorized into 2 groups, namely, the DAMA group and the non-DAMA group. We compared these groups with respect to age, gender, trauma or non-trauma status, type of hospital, health insurance, level of consciousness on admission, and diagnosis.

Results Of 8,000,529 patients, 222,389 (2.78\%) left against medical advice. The risk factors for DAMA across all age groups were as follows: no medical insurance (odds ratio [OR], 1.993), initial response to voice $(O R, 2.753)$ or pain $(O R, 2.101)$, trauma admission $(O R, 1.126)$, admission to a local emergency medical center $(O R, 1.215)$, and increased age. A high risk of DAMA was observed among patients with immune, endocrine, psychiatric, neurological, circulatory diseases, and external causes of morbidity and mortality.

Conclusion Although DAMA cases account for only a small percentage of hospital discharges, they are important because DAMA patients have high readmission and mortality rates. It is therefore important to understand the general characteristics and predictors of DAMA in order to improve patient outcome and minimize the economic burden on the healthcare system.

Keywords Organization and administration; Emergency service, hospital; Patient discharge

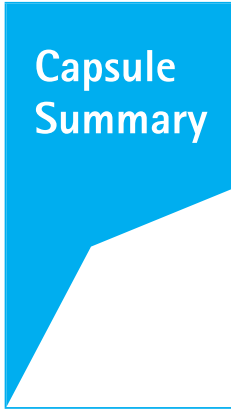

What is already known

Discharge against medical advice carries a risk of increased mortality and readmissions.

What is new in the current study

It is important to understand the general characteristics and predictors of discharge against medical advice and utilize them as a basis for improving patient outcomes and minimizing the economic burden.
Received: 6 January 2016

Revised: 23 April 2016

Accepted: 1 May 2016

Correspondence to: Choung Ah Lee Department of Emergency Medicine, Hallym University Dongtan Sacred Heart Hospital, 7 Keunjaebong-gil, Hwaseong 18450, Korea E-mail: cuccum@hanmail.net

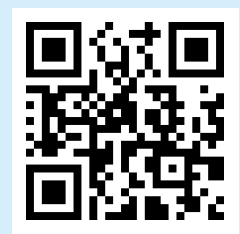

How to cite this article: Lee CA, Cho JP, Choi SC, Kim HH, Park JO. Patients who leave the emergency department against medical advice. Clin Exp Emerg Med 2016;3(2):88-94.

This is an Open Access article distributed under the terms of the Creative Commons Attribution Non-Commercial License (http:// creativecommons.org/licenses/by-nc/4.0/). 


\section{INTRODUCTION}

Emergency departments (EDs) provide acute medical care and decisions to admit or discharge a patient are made within limited time constraints. At times, patients refuse medication, further evaluation, or hospital admission against medical advice. Reasons include conflict with staff, dissatisfaction with hospital care, a past medical history of substance abuse, inability to pay hospital expenses, psychiatric problems, conflicts between carers and patients, lack of significant clinical improvement, beliefs in traditional medicine, long waiting periods, and patients' place of residence. ${ }^{1-6}$ Cases where patients leave the hospital against medical advice are referred to as discharge against medical advice (DAMA).

DAMA from the ED accounts for $0.1 \%$ to $2.7 \%$ of all discharg$\mathrm{es}^{6-9}$ and does carry potential risks. Some studies have reported that DAMA can increase the risk of mortality and of hospital readmission. ${ }^{1,4,6,10-12}$ Baptist et al. ${ }^{1}$ showed that DAMA patients with asthma had a higher risk of readmission, and Fiscella et al. ${ }^{4}$ found a high rate of mortality among DAMA patients with myocardial infarctions or unstable angina.

The human rights of patients who want to self-discharge and the obligations of doctors to continue medical care are contentious issues. ${ }^{13}$ Cost of hospitalization associated with DAMA are up to $50 \%$ greater than those where discharge is authorized by clinicians, ${ }^{14}$ and health care providers are confronted with ethical and legal difficulties, in addition to the increased burden on public health resources. ${ }^{13}$

Many studies have identified the prevalence and risks of DAMA, however, the DAMA rate remains unchanged. The aim of the present study was to determine the characteristics of patients who were discharged against medical advice from the ED in Korea to help reduce poor outcomes and the economic burden.

\section{METHODS}

\section{Design and setting}

A cross-sectional retrospective study was conducted. This study included admissions to the ED in 138 sentinel hospitals from January 2010 to December 2011. We reviewed data from the National Emergency Department Information System (NEDIS), which is controlled by the National Emergency Medical Center. The NEDIS is a computerized system that tracks the progress of ED patients from their arrival to treatment, and enables data to be updated in near-real time. ${ }^{15}$

The NEDIS dataset includes the type of hospital and patient gender, age, and level of consciousness upon arrival, type of health insurance, trauma or non-trauma status, diagnosis, and patient outcome. The hospital system is divided into 3 levels, namely, 16 regional emergency medical centers (REMCs), 118 local emergency medical centers (LEMCs), and 4 specialty care centers (SCCs). One REMC is allocated to each metropolitan city or province according to the distribution of the medical infrastructure, demographics, and population. General hospitals within a geographical area may be designated as a REMC. A LEMC is established by the governor or mayor to provide local people with access to the medical facilities and services according to the Emergency Medical Service Law. The LEMCs are distributed as follows: one center per 1 million people in metropolitan and major cities, and one center per 0.5 million people at the provincial level. A SCC status is assigned by the Minister for Health, Welfare and Family Affairs to general hospitals with the approval of governors and mayors. SCCs treat patients with trauma or burns or who are under the influence of poisonous substances. ${ }^{15}$

The DAMA cases were extracted from the disposition variables after the ED treatment. The AVPU (alert-verbal-painful-unresponsive) scale was used to determine the patient's level of consciousness. Insurance policies were classified into Medicare, Medicaid, auto insurance, workers' compensation insurance, private insurance, self-pay, and other types of insurance. Diagnoses were categorized into 22 chapters based on the international statistical classification of diseases and related health problems 10th revision (ICD-10). ${ }^{16}$ Admissions for non-medical purposes, such as the issuing of certificates and records were excluded.

\section{DAMA vs. non-DAMA patients}

Subjects were categorized into 2 groups, namely, the DAMA group and the non-DAMA group. We compared these groups with respect to age, gender, trauma or non-trauma status, type of hospital, insurance, initial level of consciousness, and diagnosis.

As SCCs are referral hospitals for specialty care, most patients visit with the thought of admission and further work up. Therefore, we did not count DAMA rates from SCCs according to hospital type. Insurance status was classified as the presence (selfpay) or absence of medical insurance. To analyze the factors affecting DAMA, subjects were stratified into the following age groups: the pediatric group (18 years or younger), the youth and middleaged group (19 to 64 years of age), and the aged group (65 years and above).

\section{Statistical analysis}

Statistical analysis was conducted using IBM SPSS ver. 19.0 (IBM Corp., Armonk, NY, USA). Descriptive analyses were performed to evaluate the general characteristics of the patients. Student's ttests were used to compare the ages between the DAMA group 
and the non-DAMA group. Chi-square tests were used to analyze categorical variables, such as patient gender, type of hospital, insurance status and initial level of consciousness. A multiple logistic regression analysis was performed to determine independent predictors of DAMA according to age group. We constructed a multivariate model using variables selected from the univariate analysis, including gender, insurance status, trauma, initial mental state, the type of hospital, and the diagnosis group by the ICD10 chapter.

\section{RESULTS}

\section{General characteristics}

A total of $8,352,652$ cases were registered in the NEDIS dataset from 2010 to 2011. Of these, 8,000,529 cases were chosen for further analysis. Patients who visited LEMCs accounted for $80.40 \%$ of the total, while those who visited REMCs accounted for $15.26 \%$. The median age was 35.15 years and, 4,264,701 (53.31\%) subjects were male. The number of patients who left against medical advice was $222,389(2.78 \%)$. The number of trauma patients was $2,219,641$, accounting for $27.74 \%$ of the total. A total of $7,750,478$
(96.87\%) patients were alert when they visited the medical center. We identified 5,577,472 (69.71\%) patients who were discharged following successful treatment in the emergency room while $1,698,129(21.23 \%)$ patients were admitted for further investigations or treatment (Table 1).

\section{Comparison between the DAMA and Non-DAMA patients} Table 2 presents the characteristics of the DAMA and non-DAMA patients. The mean age of patients in the DAMA group (43.84 \pm 23.01 years) was significantly higher than that of patients in the non-DAMA group ( $34.75 \pm 25.47$ years). The univariate analysis revealed a statistically significant correlation between the rate of DAMA and the following variables: male gender $(P<0.001)$, selfpay status $(P<0.001)$, admission to a local emergency medical center $(P<0.001)$, and trauma admission $(P<0.001)$. The male rate among DAMA patients was $54.98 \%$, which is 1.072 -fold higher than that among non-DAMA patients (53.26\%). Trauma patients self-discharged at a rate 1.126-fold higher than that of non-DAMA patients, at a rate of $30.11 \%$. There were more DAMA cases at LEMCs than at REMCs (unadjusted odds ratio [OR], 1.215). High rates of DAMA were observed among patients without medi-

Table 1. General characteristics of patients attending the emergency department between 2010 and 2011

\begin{tabular}{|c|c|c|}
\hline \multicolumn{2}{|l|}{ Characteristics } & Value \\
\hline All patients & & $8,000,529(100.00)$ \\
\hline Age $(y r)$ & & $35.00 \pm 25.45$ \\
\hline Gender & Male & $4,264,701(53.31)$ \\
\hline \multirow[t]{7}{*}{ Insurance } & National health insurance & $7,037,411(87.96)$ \\
\hline & National medical aid & $405,598(5.07)$ \\
\hline & Auto insurance & $319,753(4.00)$ \\
\hline & Workers' compensation insurance & $25,045(0.31)$ \\
\hline & Private & $2,250(0.03)$ \\
\hline & Self-pay & $165,796(2.07)$ \\
\hline & Others & $44,676(0.56)$ \\
\hline \multirow[t]{9}{*}{ Disposition } & Discharge following recovery & $5,577,472(69.71)$ \\
\hline & Discharge on grounds of medical futility & $4,293(0.05)$ \\
\hline & Other discharge & $321,484(4.02)$ \\
\hline & Transfer to other hospital for further management & $50,497(0.63)$ \\
\hline & Transfer to other hospital for conservative care & $72,013(0.90)$ \\
\hline & Transfer to other hospital for other reason & $8,286(0.10)$ \\
\hline & Hospitalization & $1,698,129(21.23)$ \\
\hline & Death & $45,966(0.57)$ \\
\hline & Discharge against medical advice & $222,389(2.78)$ \\
\hline \multirow[t]{2}{*}{ Trauma } & Disease (non-trauma) & $5,780,888(72.26)$ \\
\hline & Trauma & $2,219,641(27.74)$ \\
\hline \multirow[t]{4}{*}{ Initial level of consciousness } & Alert & 7,750,478 (96.87) \\
\hline & Responds to voice & $109,645(1.37)$ \\
\hline & Responds to pain & $78,881(0.99)$ \\
\hline & Unresponsive & $61,525(0.77)$ \\
\hline \multirow[t]{3}{*}{ Type of center } & Regional emergency medical centers & 1,221,006 (15.26) \\
\hline & Local emergency medical centers & $6,432,224(80.40)$ \\
\hline & Specialty care centers & $347,299(4.34)$ \\
\hline
\end{tabular}

Values are presented as number (\%) or mean \pm standard deviation. 
Table 2. Comparison of DAMA and non-DAMA

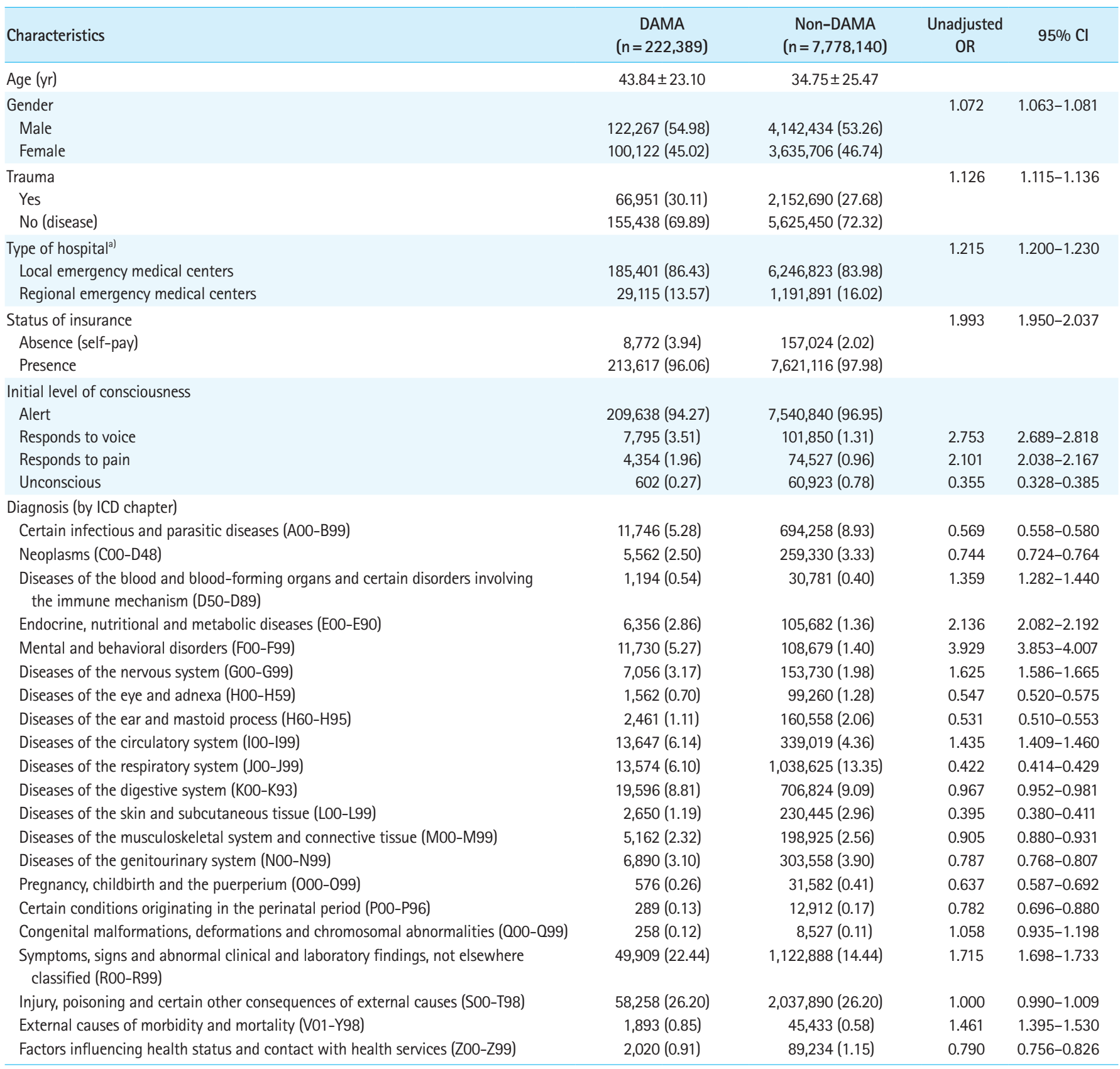

Values are presented as mean \pm standard deviation or number (\%).

DAMA, discharge against medical advice; $\mathrm{OR}$, odds ratio; $\mathrm{Cl}$, confidence interval; $I C D$, international statistical classification of diseases and related health problems.

a) Subjects from specialty care centers were excluded.

cal insurance. The DAMA rate was higher in patients who were responsive to pain or voice on admission to the ED than in those who were alert. In cases categorized as ICD-10 chapter 3, 4, 5, 6, 9,18 , and 20 diseases, the DAMA rate was significantly higher, particularly amongst patients with mental and behavioral disorders (chapter 5).

The confounding factors of gender, trauma, type of hospital, status of medical insurance, initial level of consciousness and di- agnosis group were adjusted according to age groups. Self-pay status (adjusted OR [aOR], 1.573), LEMC admission (aOR, 1.327), trauma admission $(a O R, 1.183)$, response to voice $(a O R, 1.722)$ and pain (aOR, 1.381), and ICD-10 chapter 4, 5, 6, 9, 11, 13, 14, $15,16,17,18,19,20$ diseases were predictors of DAMA in the pediatrics group. In the youth and the middle-aged group, male gender (aOR, 1.201), self-pay (aOR, 1.836), LEMC admission (aOR, 1.251), trauma admission ( $a O R, 1.307)$, initial response to voice 
Table 3. Results from multivariable modeling for predictors of discharge against medical advice by age group

\begin{tabular}{|c|c|c|c|c|c|c|}
\hline \multirow[t]{2}{*}{ Characteristics } & \multicolumn{2}{|c|}{$\begin{array}{l}\text { The pediatric group } \\
\qquad(n=2,481,727)\end{array}$} & \multicolumn{2}{|c|}{$\begin{array}{l}\text { The youth and the } \\
\text { middle-aged group } \\
(n=3,968,009)\end{array}$} & \multicolumn{2}{|c|}{$\begin{array}{l}\text { The aged group } \\
(n=1,203,494)\end{array}$} \\
\hline & $\begin{array}{l}\text { Adjusted } \\
\text { OR }\end{array}$ & $95 \% \mathrm{Cl}$ & $\begin{array}{l}\text { Adjusted } \\
\text { OR }\end{array}$ & $95 \% \mathrm{Cl}$ & $\begin{array}{l}\text { Adjusted } \\
\text { OR }\end{array}$ & $95 \% \mathrm{Cl}$ \\
\hline Gender, male & 1.014 & $0.992-1.037$ & 1.201 & $1.188-1.215$ & 1.093 & $1.073-1.114$ \\
\hline Self-pay & 1.573 & $1.457-1.698$ & 1.836 & $1.790-1.883$ & 1.041 & $0.959-1.130$ \\
\hline Local emergency medical centers & 1.327 & $1.284-1.372$ & 1.251 & $1.231-1.271$ & 1.241 & $1.210-1.273$ \\
\hline Trauma (non-disease) & 1.183 & $1.128-1.241$ & 1.307 & $1.280-1.333$ & 1.098 & $1.055-1.144$ \\
\hline \multicolumn{7}{|l|}{ Initial level of consciousness, alert } \\
\hline Responds to voice & 1.722 & $1.533-1.934$ & 2.272 & $2.201-2.345$ & 1.262 & $1.210-1.316$ \\
\hline Responds to pain & 1.381 & $1.197-1.592$ & 1.569 & $1.504-1.638$ & 1.060 & $1.006-1.117$ \\
\hline Unconscious & 0.420 & $0.287-0.615$ & 0.269 & $0.242-0.300$ & 0.150 & $0.132-0.171$ \\
\hline \multicolumn{7}{|l|}{ Diagnosis } \\
\hline Certain infectious and parasitic diseases (A00-B99) & 1.022 & $0.902-1.157$ & 0.911 & $0.859-0.965$ & 1.018 & $0.891-1.164$ \\
\hline Neoplasms (C00-D48) & 0.955 & $0.751-1.214$ & 0.864 & $0.809-0.922$ & 0.919 & $0.807-1.048$ \\
\hline $\begin{array}{l}\text { Diseases of the blood and blood-forming organs and certain disorders involving the } \\
\text { immune mechanism (D50-D89) }\end{array}$ & 1.218 & $0.964-1.539$ & 1.820 & $1.649-2.009$ & 2.119 & $1.813-2.476$ \\
\hline Endocrine, nutritional and metabolic diseases (E00-E90) & 1.739 & $1.379-2.193$ & 2.238 & $2.095-2.390$ & 2.256 & $1.982-2.567$ \\
\hline Mental and behavioral disorders (F00-F99) & 8.577 & $7.445-9.881$ & 4.085 & $3.859-4.324$ & 2.303 & $2.006-2.642$ \\
\hline Diseases of the nervous system (G00-G99) & 3.474 & $3.029-3.983$ & 1.805 & $1.698-1.919$ & 1.837 & $1.606-2.101$ \\
\hline Diseases of the eye and adnexa (H0O-H59) & 1.021 & $0.861-1.212$ & 0.593 & $0.547-0.642$ & 0.904 & $0.748-1.093$ \\
\hline Diseases of the ear and mastoid process ( $\mathrm{H} 60-\mathrm{H} 95)$ & 0.689 & $0.594-0.800$ & 0.848 & $0.786-0.915$ & 1.066 & $0.919-1.236$ \\
\hline Diseases of the circulatory system (I00-199) & 2.748 & $2.319-3.257$ & 1.692 & $1.597-1.794$ & 1.377 & $1.213-1.564$ \\
\hline Diseases of the respiratory system (J00-J99) & 0.878 & $0.777-0.993$ & 0.709 & $0.668-0.753$ & 1.168 & $1.026-1.331$ \\
\hline Diseases of the digestive system (K00-K93) & 1.153 & $1.014-1.311$ & 1.316 & $1.245-1.391$ & 1.079 & $0.949-1.227$ \\
\hline Diseases of the skin and subcutaneous tissue (L00-L99) & 0.825 & $0.717-0.949$ & 0.523 & $0.487-0.562$ & 0.720 & $0.606-0.855$ \\
\hline Diseases of the musculoskeletal system and connective tissue (M00-M99) & 1.582 & $1.360-1.840$ & 0.958 & $0.900-1.019$ & 1.171 & $1.021-1.343$ \\
\hline Diseases of the genitourinary system (N00-N99) & 1.544 & $1.329-1.795$ & 0.915 & $0.862-0.972$ & 0.966 & $0.844-1.105$ \\
\hline Pregnancy, childbirth and the puerperium (000-099) & 3.005 & $1.935-4.670$ & 0.777 & $0.703-0.860$ & 1.548 & $0.754-3.175$ \\
\hline Certain conditions originating in the perinatal period (P00-P96) & 2.141 & $1.793-2.557$ & 1.143 & $0.791-1.652$ & 2.342 & $1.544-3.551$ \\
\hline Congenital malformations, deformations and chromosomal abnormalities (000-099) & 3.727 & $3.044-4.562$ & 1.138 & $0.909-1.425$ & 0.950 & $0.585-1.542$ \\
\hline $\begin{array}{l}\text { Symptoms, signs and abnormal clinical and laboratory findings, not elsewhere classi- } \\
\text { fied (R00-R99) }\end{array}$ & 2.237 & $1.981-2.527$ & 1.976 & $1.872-2.086$ & 2.060 & $1.817-2.337$ \\
\hline Injury, poisoning and certain other consequences of external causes (S00-T98) & 1.421 & $1.257-1.607$ & 1.013 & $0.960-1.070$ & 1.200 & $1.055-1.365$ \\
\hline External causes of morbidity and mortality (V01-Y98) & 1.343 & $1.095-1.648$ & 1.447 & $1.343-1.558$ & 1.336 & $1.109-1.611$ \\
\hline
\end{tabular}

$\mathrm{OR}$, odds ratio; $\mathrm{Cl}$, confidence interval.

$(\mathrm{aOR}, 2.272)$ and pain $(\mathrm{aOR}, 1.569)$, and ICD-10 chapter $3,4,5,6$, $9,11,18,20$ diseases were found to be predictors of DAMA. Among the aged group, male gender (aOR, 1.093), LEMC admission (aOR, 1.241), trauma admission ( $a O R, 1.098)$, initial response to voice $(\mathrm{aOR}, 1.262)$ and pain $(\mathrm{aOR}, 1.060)$, and ICD-10 chapter $3,4,5,6$, $9,10,13,18,19,20$ diseases were found to be predictors of DAMA (Table 3).

\section{DISCUSSION}

Patient complaints about poor communication with health professionals or the high costs of hospitalization are well recognized in clinical practice. Some patients believe that their health improves following admission to hospital, whereas others are dissatisfied with their treatment. For a number of reasons, patients return home against medical advice despite the high risks of readmission and mortality. The DAMA rate in EDs is approximately $1 \%$ to $2 \%$ of admissions, which is similar to the DAMA rate in hospitalized patients. ${ }^{3,8,17-20}$

The current study reveals that the incidence of DAMA and the higher prevalence among males are comparable to statistics reported in the literature. The mean age of DAMA patients in Korea (43.84 years) was similar to that in other countries; however, the mean age of non-DAMA patients was significantly younger than that in previous reports. ${ }^{3,13,18,21}$

Subjects were divided into three groups according to their age. The discharge of pediatric patients is usually determined by their parents, while in aged patients, discharge is generally influenced by their caregivers. Accordingly, DAMA factors were analyzed according to age group. 
Trauma, hospital type, status of insurance, and initial level of consciousness influenced DAMA rates across all age groups. The current study showed that the DAMA rate among patients admitted with trauma was higher than that among patients with nontraumatic medical conditions. Many studies have indicated that alcohol or drug abuse is a predictor of both DAMA and injury. ${ }^{22}$ DAMA in injured patients is thought to be influenced by alcohol consumption. Duffy ${ }^{23}$ found that patients admitted to rural hospitals were more likely to leave against medical advice than patients admitted to urban hospitals. This is consistent with our finding that the DAMA rate in LEMCs was higher than that in REMCs as every REMC in Korea is located in an urban area. The inability to pay for hospital expenses is one important reason for leaving an ED and is the most common cause of the high DAMA rate among patients without insurance. 6,21,24,25 There were more DAMA cases among patients who responded to verbal or painful stimuli compared with those who were mentally alert. We speculate that people under the influence of alcohol or drugs became conscious as time passed.

An important characteristic of this study is that we analyzed the distribution of DAMA cases according to the ICD-10 diagnosis codes. In ICD-10 chapters 4, 5, 6, 9, 18, and 20, the DAMA ratio was high in all age groups. Chapters 4 and 6 were endocrine, nutritional, and metabolic diseases (ICD-10 code, E00-E90) and diseases of the nervous system (ICD-10 code, with G00-G99). We postulated that patients with chronic diseases refused to remain in hospital for a long time. Patients with mental and behavioral disorders (ICD-10 code, F00-F99; chapter 5) had the highest risk of DAMA, which is consistent with previous studies. ${ }^{9}$ In the pediatric group, the DAMA rate was 8.577 times higher, which was significantly high. Additionally, diseases of the circulatory system (ICD-10 code, I00-I99; chapter 9) were associated with a high risk of DAMA, consistent with previous studies. ${ }^{4}$

Interestingly, chapter 18 (symptoms, signs and abnormal clinical and laboratory findings, not elsewhere classified; ICD-10 code, R00-R99) and chapter 20 (external causes of morbidity and mortality; ICD-10 code, V01-Y98) were also associated with a high risk of DAMA. The variable of diagnoses is based on the first impression by physicians. Sometimes physicians record patients' diagnoses before they have completed work-ups. In these cases, ICD codes are recorded as R00-R00 or V01-Y98 based only on symptoms, signs, and injury causes. According to one study, intentional injuries (ICD 10 code, X60-Y34) are closely associated with DAMA ${ }^{26}$ Complications of medical and surgical care (ICD 10 code, Y40-Y84) influence patient satisfaction with treatment. We postulated that the DAMA rate in ICD-10 chapter 20 is related to injuries and complications.

Some ICD-10 chapter groups showed high DAMA rates in a specific age group, not in all age groups. The aged group with diseases of the respiratory system (ICD-10 code, J00-J99; chapter 10) showed a high DAMA rate $(a O R, 1.168)$ compared to the pediatric and the youth and middle-aged groups. This is because many aged patients have chronic respiratory disease which, as with other chronic conditions, is associated with an increased DAMA rate. The DAMA rate in the pediatric patients with congenital malformations, deformities and chromosomal abnormalities (ICD-10 code, 000-099; chapter 17) is high (aOR, 3.727) because parents who have taken care of their infants for a long period of time are less likely to comply with doctors' directions.

This study has the strength of a large-scale investigation overseen by a national organization. We successfully obtained objective data minimizing any bias associated with single center studies. Nonetheless, this study has two main limitations. Firstly, it identifies patients who left the ED against medical advice on the basis of a final diagnosis and a limited set of administrative data. We need more information to explain why patients leave, how DAMA can be prevented, and whether there are any negative outcomes for these patients. Secondly, we used only one primary diagnosis, even though patients may have two or more diagnoses. For example, trauma injuries can be classified according to the mechanism of injury or its effect. Our registry did not prioritize between mechanisms and results. Therefore, this study showed that chapter 19 diagnoses (ICD-10 code, S00-T98) are not related to DAMA, while trauma was a predictor of DAMA.

Furthermore, the chapter classification based on the ICD-10 code is not specific enough for individual diseases. A more detailed classification by disease may be necessary to further characterize DAMA factors. Finally, to predict DAMA using our logistic regression analysis model, external validation with the NEDIS dataset, over another year, is required.

In conclusion, we identified the general characteristics of DAMA patients and differences between the DAMA and non-DAMA groups. The risk factors for DAMA across all age groups were self-pay status (no insurance), response to voice or pain, trauma, admission at a local emergency medical center, and older age. According to the ICD-10 disease groups, there is a high risk of DAMA among patients who have immune, endocrine, psychiatric, neurological, circulatory diseases, and external causes of morbidity and mortality. This study will be utilized as a basis for improving patient outcomes and minimizing the economic burden.

\section{CONFLICT OF INTEREST}

No potential conflict of interest relevant to this article was reported. 


\section{ACKNOWLEDGMENTS}

We would like to thank the National Emergency Medical Center for providing the data in this study.

\section{REFERENCES}

1. Baptist AP, Warrier I, Arora R, Ager J, Massanari RM. Hospitalized patients with asthma who leave against medical advice: characteristics, reasons, and outcomes. J Allergy Clin Immunol 2007;119:924-9.

2. Ibekwe RC, Muoneke VU, Nnebe-Agumadu UH, Amadife MA. Factors influencincing discharge against medical advice among paediatric patients in Abakaliki, Southeastern Nigeria. J Trop Pediatr 2009;55:39-41.

3. Ibrahim SA, Kwoh CK, Krishnan E. Factors associated with patients who leave acute-care hospitals against medical advice. Am J Public Health 2007;97:2204-8.

4. Fiscella K, Meldrum S, Barnett S. Hospital discharge against advice after myocardial infarction: deaths and readmissions. Am J Med 2007;120:1047-53.

5. Anis AH, Sun H, Guh DP, Palepu A, Schechter MT, O'Shaughnessy MV. Leaving hospital against medical advice among HIV-positive patients. CMAJ 2002;167:633-7.

6. Ding R, Jung JJ, Kirsch TD, Levy F, McCarthy ML. Uncompleted emergency department care: patients who leave against medical advice. Acad Emerg Med 2007;14:870-6.

7. Dubow D, Propp D, Narasimhan K. Emergency department discharges against medical advice. J Emerg Med 1992;10:513-6.

8. Monico EP, Schwartz I. Leaving against medical advice: facing the issue in the emergency department. J Healthc Risk Manag 2009;29:6-9,13,15.

9. Brook M, Hilty DM, Liu W, Hu R, Frye MA. Discharge against medical advice from inpatient psychiatric treatment: a literature review. Psychiatr Serv 2006;57:1192-8.

10. Southern WN, Nahvi S, Arnsten JH. Increased risk of mortality and readmission among patients discharged against medical advice. Am J Med 2012;125:594-602.

11. Hwang SW, Li J, Gupta R, Chien V, Martin RE. What happens to patients who leave hospital against medical advice? CMAJ 2003;168:417-20.

12. Glasgow JM, Vaughn-Sarrazin $M$, Kaboli PJ. Leaving against medical advice (AMA): risk of 30-day mortality and hospital readmission. J Gen Intern Med 2010;25:926-9.
13. Alfandre DJ. "I'm going home": discharges against medical advice. Mayo Clin Proc 2009;84:255-60.

14. Aliyu ZY. Discharge against medical advice: sociodemographic, clinical and financial perspectives. Int J Clin Pract 2002; 56:325-7.

15. National Emergency Medicine Center. National Emergency Department Information System [Internet]. Seoul: National Emergency Medicine Center [cited 2016 Apr 26]. Available from: http://www.nemc.or.kr/egen/inf.emb21.do.

16. World Health Organization. International statistical classification of diseases and related health problems: 10th revision. 2010 ed. Geneva: World Health Organization; 2010.

17. Garland A, Ramsey $C D$, Fransoo $R$, et al. Rates of readmission and death associated with leaving hospital against medical advice: a population-based study. CMAJ 2013;185:1207-14.

18. Yong TY, Fok JS, Hakendorf $\mathrm{P}$, Ben-Tovim D, Thompson $\mathrm{CH}$, Li JY. Characteristics and outcomes of discharges against medical advice among hospitalised patients. Intern Med J 2013; 43:798-802.

19. Devitt PJ, Devitt AC, Dewan M. Does identifying a discharge as "against medical advice" confer legal protection? J Fam Pract 2000;49:224-7.

20. O'Hara D, Hart W, McDonald I. Leaving hospital against medical advice. J Qual Clin Pract 1996;16:157-64.

21. Levy $F$, Mareiniss DP, lacovelli $C$. The importance of a proper against-medical-advice (AMA) discharge: how signing out AMA may create significant liability protection for providers. J Emerg Med 2012;43:516-20.

22. Cherpitel CJ, Ye Y, Watters K, Brubacher JR, Stenstrom R. Risk of injury from alcohol and drug use in the emergency department: a case-crossover study. Drug Alcohol Rev 2012;31:431-8.

23. Duffy D. Discharged against medical advice: causes and consequences. Can Fam Physician 1990;36:1495-8.

24. Kim SW, Kim OJ, Jang SJ, Jung KY, Kim SH. The analysis of discharge against medical advice in the emergency department. J Korean Soc Emerg Med 1993;4:116-22.

25. Magauran BG Jr. Risk management for the emergency physician: competency and decision-making capacity, informed consent, and refusal of care against medical advice. Emerg Med Clin North Am 2009;27:605-14.

26. Lee $\mathrm{CA}$, Choi SC, Jung KY, et al. Characteristics of patients who visit the emergency department with self-inflicted injury. J Korean Med Sci 2012;27:307-12. 